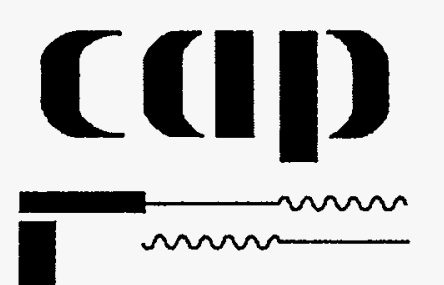

BNL- 64108

CAP-157-96C

\title{
RECENT PROGRESS ON PHOTONIC BAND GAP ACCELERATOR
} CAVITIES

\author{
D.R. Smith, Derun Li, D.C. Vier, N. Kroll, S. Schultz \\ Department of physics \\ University of California, San Diego \\ La Jolla, CA 92093-0319
}

H. Wang

Department of Physics

Brookhaven National Laboratory Upton, NY 11973

February 1997

PFC.FIVET)

MAR 251937

OSTI

DISTRIBUTION OF THIS DOCIMENT IS UMIMATEP

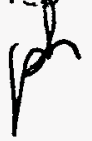

\section{CENTER FOR ACCELERATOR PHYSICS}




\title{
Recent Progress on Photonic Band Gap Accelerator Cavities
}

\author{
D. R. Smith*, Derun Li*, D. C. Vier*, N. Kroll*i, S. Schultz* \\ *Department of Physics, University of Califomia, San Diego \\ 9500 Gilman Drive, Lałyolla, California $92093-0319$ \\ †Stanford Lineár Accelerator Center, Stanford, CA 94305
}

\author{
H. Wang \\ Center for Accelerator Physics, Brookhaven National Laboratory \\ Upton, New York, 11973
}

\begin{abstract}
We report on the current status of our program to apply Photonic Band Gap (PBG) concepts to produce novel high-energy, high-intensity accelerator cavities. The PBG design on which we have concentrated our inital efforts consists of a square array of metal cylinders, terminated by conducting or superconducting sheets, and surrounded by microwave absorber on the periphery of the structure. A removed cylinder from the center of the array constitutes a site defect where a localized electromagnetic mode can occur. In previous work, we have proposed that this structure could be utilized as an accelerator cavity, with advantageous properties over conventional cavity designs. In the present work, we present further studies, including MAFIAbased numerical calculations and experimental measurements, demonstrating the feasibility of using the proposed structure in a real accelerator application.
\end{abstract}

\section{INTRODUCTION}

A realization of a Photonic Band Gap (PBG) cavity, depicted in Figure 1, consists of a periodic lattice of conducting, or superconducting, cylinders bounded on top and bottom by conducting (or again, superconducting) plates. Removing a cylinder from the center of the array forms a defect site, where a localized electromagnetic accelerating mode can exist over a wide range of lattice parameters. The interior lattice is surrounded by absorbing material at the periphery of the structure. For some applications, it may be desired to substitute dielectric cylinders in place of some or all of the metal cylinders; the use of dielectrics in PBG cavities will be discussed below, and also in a companion paper in these proceedings. In Figure 1 we have presented the absorber geometry as a toothed structure surrounding the periphery; but this is only one of many possible designs that might be used for higher order mode damping.

In a previous paper (1), we introduced the general concept of the PBG cavity, and listed its potential advantages over presently-used cavities. The most fundamental 
difference between the PBG cavity and a typical metal-walled cavity is with respect to the mode spectrum. A traditional cavity (e. g., a "pill-box" cavity),

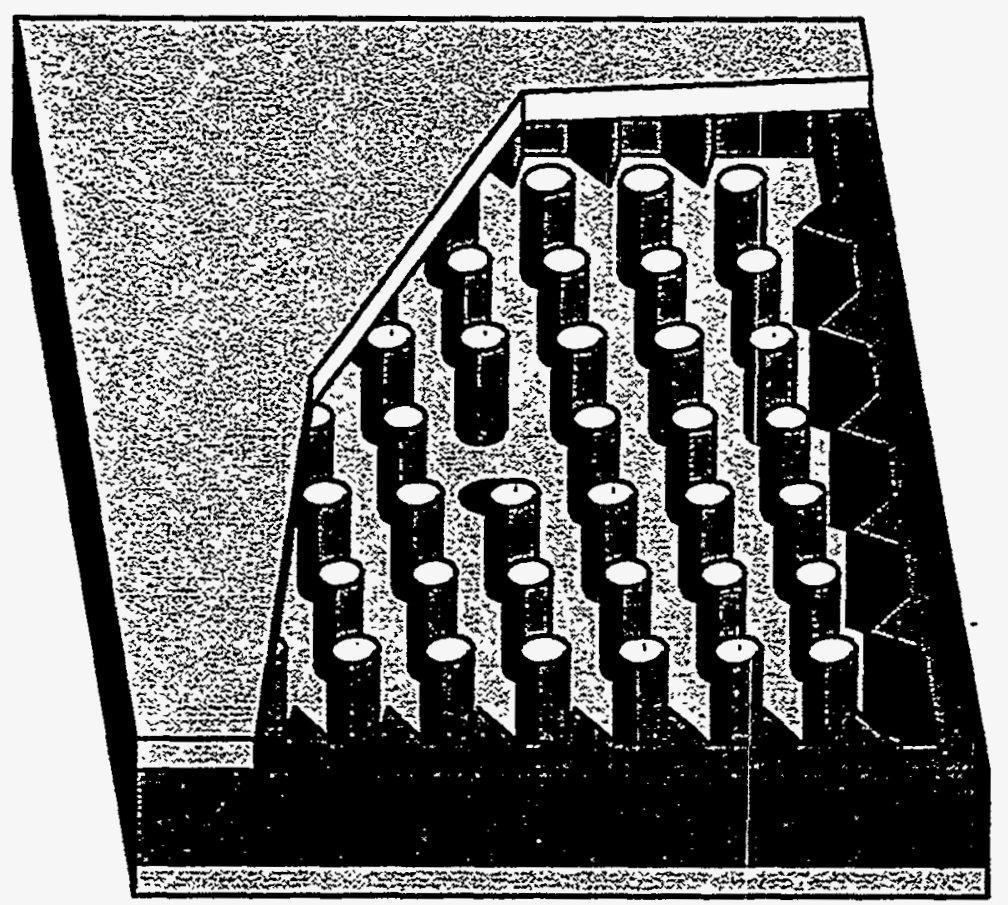

FGURE 1. A schematic diagram of the components of a metal PBG cavity. The spacing between cylinders is $d$, the radius of a cylinder is $r$, and the height of a cylinder is $h$. Microwave absorber is placed around the periphery of the structure. In our copper test unit, the cylinders were brazed into the bounding metal plates; while in the niobium unit, the cylinders were e-beam welded to the plates.

has a discrete spectrum of modes, beginning with the fundamental and continuing over all frequencies. The type of mode and mode spacing are fixed by the size and geometry of the cavity. It is generally true that over the frequency range of interest for accelerator cavities, there will exist many higher order modes (HOMs) in addition to the accelerating mode, which may be excited by a beam which passes through the cavity. The loss of energy by a beam to the HOMs of an accelerating cavity is an important figure of merit of a cavity or cavity structure, and a significant amount of effort has been expended to design structures that either minimize HOM excitation or detune HOMs $(2,3)$.

In contrast to the traditional cavity, the mode spectrum of a PBG cavity exhibits dense frequency bands of "extended" modes (known as "pass bands" in analogy with solid-state physics), interspersed with frequency bands of very low mode density (known as "stop bands"). In an infinite photonic lattice (no defects or termination), no modes can exist with frequencies in the stop bands. When the lattice contains a defect, such as the removed cylinder in the structures we consider here, "defect" modes can exist within the stop bands with fields localized at the defect site, and decaying exponentially away in all directions. In contrast, modes occuring within the pass bands have fields that are extended throughout the lattice, and can be damped by placing absorber around the 


\section{DISCLAIMIER}

Portions of this document may be illegible in electronic image products. Images are produced from the best available original document. 
periphery of the lattice, as shown in Figure 1. Since defect modes are localized at the center of the lattice, they remain relatively unperturbed by the absorber. Thus, the PBG cavity, which includes a photonic lattice surrounded by absorber, can be expected to have relatively few significant HOMs as compared to a traditional cavity.

In Figure 2 we present schematically the TM mode structures corresponding to a conventional metal-walled cavity, a dielectric-based PBG cavity, and a metal-based PBG cavity. The dielectric structure in general has periodically occuring stop bands that may or may not contain defect modes, depending on the parameters of the lattice. Also, it is a feature of dielectric based structures that the lowest modes (from zero frequency) are extended, and form the first pass band. The third part of the diagram shows schematically the TM modes of the particular metal PBG cavity we have been investigating. There is only one bound mode, which occurs as the lowest-frequency mode of the cavity.

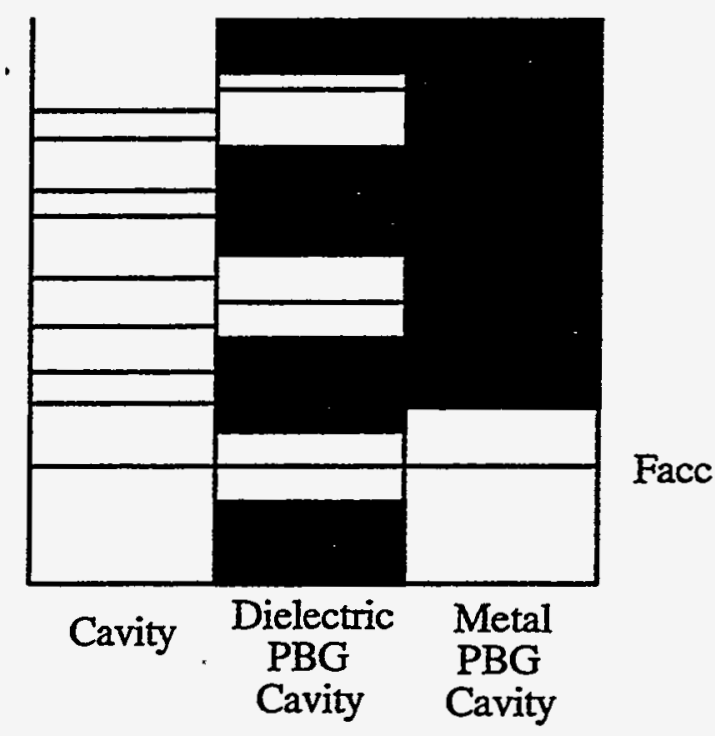

FIGURE 2. A conceptual diagram comparing the TM modes of a conventional cavity, a dielectric PBG cavity, and of a particular metal PBG cavity. For some parameters of a/d the metal PBG will have frequency band gaps in addition to the lowest; however, for the parameters we actually use, the diagram is accurate.

\section{SUMMARY OF ACCELERATING MODE PROPERTIES}

\section{Q of the Accelerating Mode}

The field pattern of the defect mode of the metal PBG cavity is very similar to that of the $\mathrm{TM}_{010}$ mode of a pill-box cavity. The mode has monopole symmetry, with a field maximum at the center of the defect region. The field decays into the surrounding photonic lattice, with an energy $\left(\mathrm{E}^{2}\right)$ decay rate of roughly two orders of magnitude per lattice constant; thus, the mode is very well localized. Our initial calculations indicated that a superconducting cavity with a $9 \times 9$ photonic lattice would have a $Q$-factor limited 
by superconductor losses rather than losses at the periphery of the structure. Therefore, absorber placed at the edges of the lattice to damp HOMs should not impact the fundamental.

Since our earlier numerical methods did not have the capabilities to simulate systems with lossy materials in a realistic geometry, we began simulating the configurations with MAFIA, a commercial finite-difference electromagnetic mode solver. With MAFIA, the presence of any losses in the simulated configuration can be solved by the use of MAFIA time-domain code, in which a source excites the cavity and the fields are calculated at successive time increments. To find the $Q$ for a given configuration, we can excite the cavity at a given time $t=0$ with a current pulse and observe the subsequent time decay of the field at a some point in the cavity. Or, we can excite a continuous sinusoidally varying current density at the cavity's resonant frequency and note the field at which the solution is steady state; this field will be related to the $\mathrm{Q}$ of the mode.

For most parameters of interest, however, the $Q$ of the fundamental is so large that it is impractical to run MAFIA until we obtain a either an accurate value for the decay time, or until the steady-state value of field is reached. For Q's of this size, we generally restrict ourselves to calculating the system only during the period where the fields are increasing linearly with time, as in Figure 3 . Thus at any point during the time series, we can calculate the stored energy from the field data, the dissipated power in the regions with complex dielectric functions, and therefore the $\mathrm{Q}$ corresponding to the configuration. The $Q$ calculated for the PBG cavity simulated in Figure 3 was $~ 22,000$.

We find it convenient to calculate the $Q$ at several places during the time of linear build-up, since the contribution from the excitation source near-fields will

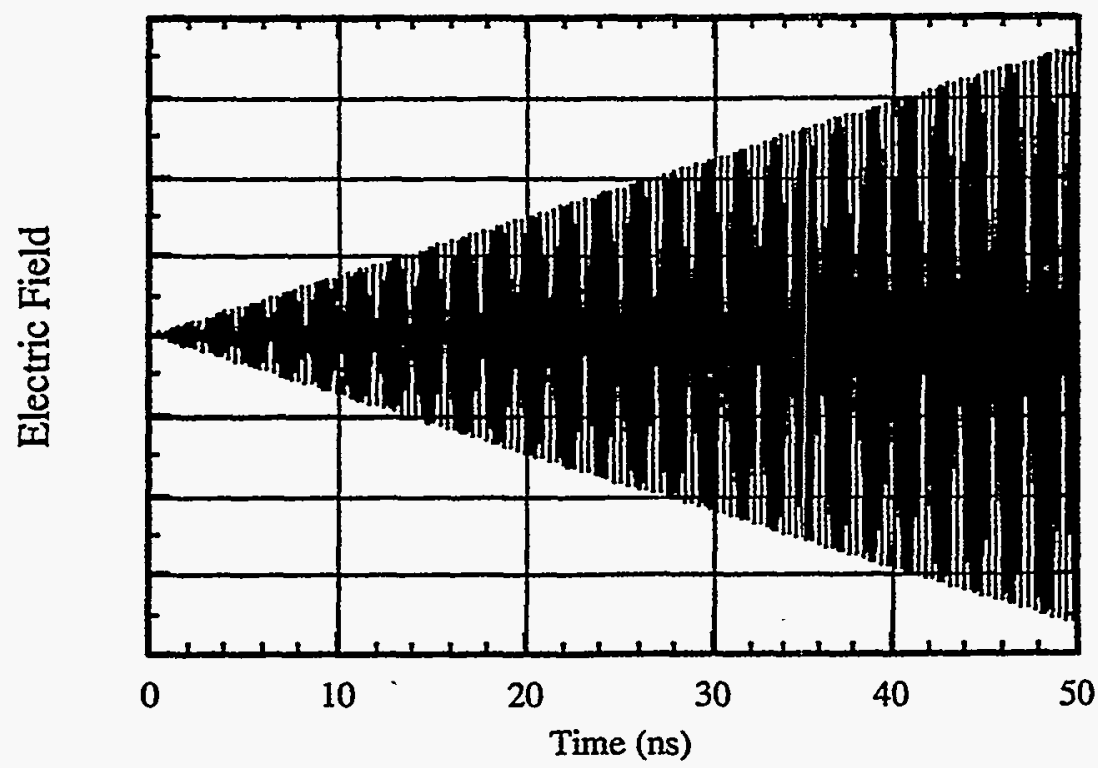

FIGURE 3. Electric field versus time at the center of a $7 \times 7$ PBG cavity. The cavity was driven by a sinusoidally varying current density at the frequency of the fundamental. The cavity 
was surrounded by a layer of toothed absorber (as shown schematically in Figure 1), which had a dielectric constant of 22 and conductivity $\sigma=0.3$. The $Q$ at $35 \mathrm{~ns}$ was calculated as 22,367 , while at $45 \mathrm{~ns}$ the $\mathrm{Q}$ was calculated as 21,918 .

inevitably contribute to the stored energy. These near-fields are bounded in time, however, and thus their relative contribution to the stored energy decreases as a function of time. If we calculate approximately the same value for the $Q$ at several times, then we can be confident that the source fields are not significantly perturbing the result.

\section{$\quad$ Effects of Introducing an Iris}

In order to make reasonable predictions about the performance of the accelerating mode in a practical structure, it is necessary to include the effect of a beam hole in the simulations. The presence of the beam hole complicates the numerical problem considerably, due to the loss of translational symmetry along the beam axis. However, MAFIA can accommodate most of the three dimensional geometries of current interest to us. In Figure 4, for example, we present a calculation of the accelerating mode frequency versus phase advance across a cell. The solid points on the curve were computed by MAFIA, for a single cell of a $7 \times 7$ PBG cavity, while the curve indicates a best fit to the conventional single band dispersion relation

$$
\left(\frac{1}{f}\right)^{2}=\left(\frac{1}{F}\right)^{2}[1+\eta \cos \varphi]
$$

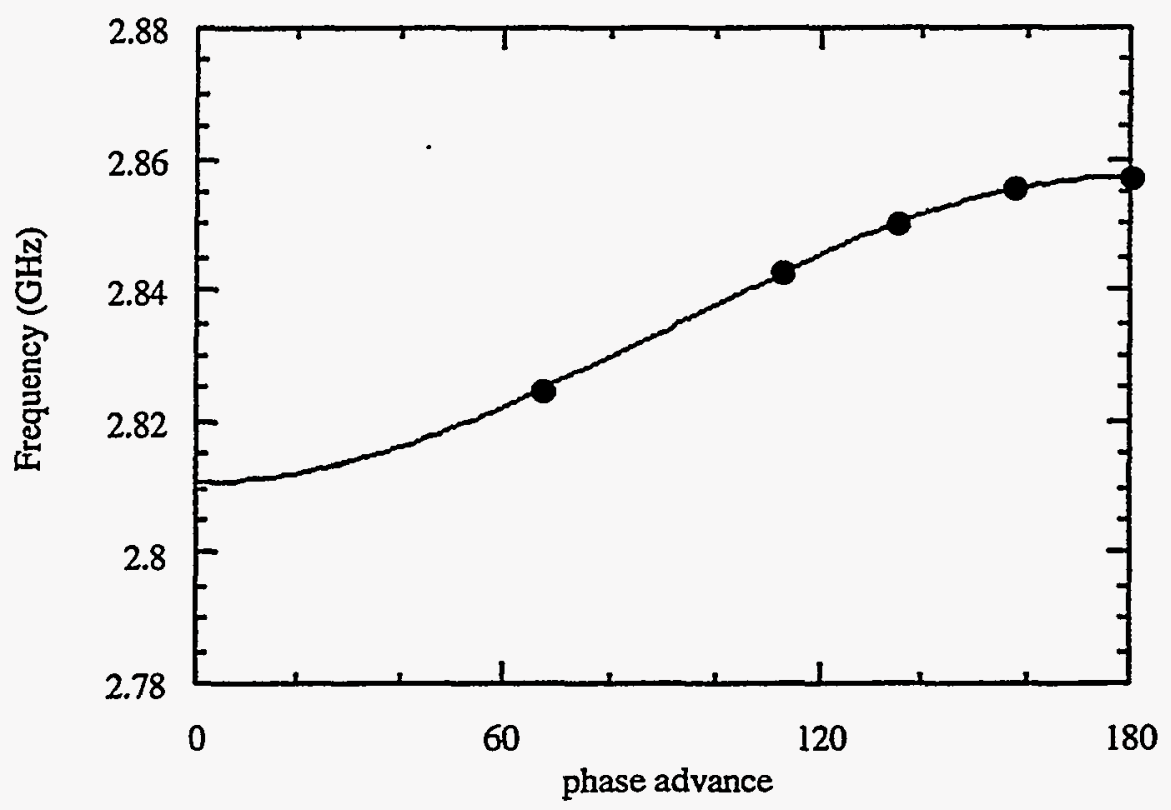

FIGURE 4. Dispersion curve of a $7 \times 7$ PBG lattice with beam holes. The parameters of the cavity are the same as those described in Figure 5. 
The best fit was obtained for the values $F=2.83372 \mathrm{GHz}$ and $\eta=0.0163591$. The value of $\eta$ is similar to conventional accelerator cavities, and confirms that we can achieve cell-tocell coupling of PBG cavities through a beam iris.

In Figure 5 we present a summary of the parameters of the accelerating mode of a 7x7 PBG cavity, calculated using MAFIA frequency-domain code. The spacing between cylinders was $4.2 \mathrm{~cm}$, while the cylinder radii $a=0.7875 \mathrm{~cm}$, for an a/d ratio of 0.1875 . For the purposes of this calculation, beam holes were included, with radius $\mathrm{mb}$ of $1.5919 \mathrm{~cm}$; centered at the defect site on both the upper and lower plates of the PBG cavity. The beam hole size was chosen arbitrarily, and matches the scaled beam-hole radius of the TESLA cavity. Since both $E_{\text {peak }} / E_{a c c}$ and the shunt impedance $R$ depend on the characteristics of the beam hole, it should be possible to significantly improve these parameters relative to the TESLA cavity. The PBG cavity length was determined as $\mathrm{L}=2.6242 \mathrm{~cm}$, with $\pi$-phase advance across the cavity.

\section{Effect of Cavity Asymmetry}

As evidenced in Figure 5, the parameters of the PBG cavity are very similar to those of presently-used accelerator cavities, which is not surprising considering that the fields are very similar to one another (particularly near the beam axis). However, the unique symmetry and geometry of the PBG suggest that some complications might arise.

For example, since the PBG cavity has a definite four-fold symmetry with respect to rotations, the field of the accelerating mode will have higher multipole components. This is in contrast to the pill-box cavity and other cylindrically symmetric structures. One might anticipate some detrimental effect on a beam which passes through such an asymmetric cavity. In order to obtain some estimate of this effect (without resorting to full beam-dynamics simulations), we can calculate the change in energy of an accelerated test particle as a function of position within the beam hole region.

The energy gain per charge is the integral:

$$
|\Delta E|=\left|\int_{0}^{d} E_{z}(r, \varphi, z) e^{i \frac{\omega z}{c}} d z\right|
$$

where $E_{2}$ is the component of the electric field in the beam direction, and the integral is taken along a line from one end of the cavity to the other, at a radial position within the beam hole. Due to the four-fold symmetry of our structure, $\Delta \mathrm{E}$ can be expanded in terms of spatial harmonics as

$$
\Delta \mathrm{E}(\mathrm{r}, \varphi) \cong \mathrm{E}_{0}\left\{+\mathrm{a}_{1} \cos (4 \varphi) \mathrm{r}^{4}+\mathrm{a}_{2} \cos (8 \varphi) \mathrm{r}^{8}\right\}
$$

with only the first three terms of the expansion retained. By taking two values of the field at $\varphi=0_{\text {_ }}$ and $\varphi=45_{\text {_ }}$ (constant $r$ ), and assuming all other orders are negligible, we can 
determine the values of the coefficients of $a_{1}$ and $a_{2}$. Using data from a MAFIA E-code calculation, we find the approximate values $a_{1}=-7.5 \times 10^{-5}(1 \pm 0.016) \mathrm{mm}^{-4}$, and $a_{2}=1.614 \times 10^{-8}(1 \pm 0.283) \mathrm{mm}^{-8}$. As an example, if we take 100 microns for the beam excursion away from the beam axis, then we obtain maximum voltage variations at the $10^{-8}$ level. This excursion distance is a reasonable beam excursion for the NLC, and thus the asymmetry effect is quite negligible.

\section{Effect of Cylinder Misalignment}

Another potential concern is the effect of slight cylinder misalignment on the accelerating mode of the PBG cavity. Typically, we find that the PBG effect is extremely robust, and relatively insensitive to minor random perturbations in the underlying photonic lattice; however, for accelerator applications, even minor results may have significance. In order to assess the effect of randomness on the mode, we simulated (using MAFIA E-code) a 7×7 PBG cavity, using lossless cylinders, and surrounded by copper walls (rather than absorber) on the periphery.

A structure with no cylinder misalignment yields a $Q$ of over $10^{11}$; for the structure in which cylinders are randomly perturbed, we expect this $Q$ to be degraded. We also expect a frequency shift. In Table 1 we see that even a sizable amount of disorder produces only minor frequency shifts and Q-degradation of the fundamental mode. The cylinder displacement used in the calculations, $\pm 1.47 \mathrm{~mm}$, was much larger than the achievable tolerance for fabrication.

It should be pointed out that the fundamental mode depends most strongly on the cylinders closest to the defect region. Since the mode decays exponentially away from the center, equivalent displacements in cylinder positions located farther from the center can be expected to produce smaller perturbations to the localized modes. This further suggests that material and fabrication precision constraints are highest immediately at the center of the structure, and are reduced away from the central group of cylinders. As an illustration of this effect, we have simulated a 9x9 PBG cavity in which we have inserted a cut in the bounding plates around the inner $7 \times 7$ photonic lattice. The cut entirely isolates the center defect and (lossless) cylinders from the outer cylinder layer and absorber. Field plots of the defect mode display no significant perturbation after the cut. Furthermore, the $Q$ of the mode is also not significantly reduced. An actual superconducting cavity constructed with a cut would only require the center portion to be cooled to cryogenic temperatures; the thermally isolated outer cylinders, absorber, and walls could be maintained at higher temperatures.

\begin{tabular}{|l|l|l|}
\hline & $\Delta \mathrm{f}(\mathrm{MHz})$ & $\mathrm{Q}$-factor $\left(\mathrm{x} \mathrm{10}^{11}\right)$ \\
\hline \hline no misalignment & 0 & 2.185 \\
\hline misalignment 1 & 6.033 & 1.74269 \\
\hline misalignment 2 & 8.536 & 1.841 \\
\hline misalignment 3 & 58.75 & 1.117 \\
\hline
\end{tabular}

TABLE 1. Effect of disorder on the frequency and $Q$ of the accelerating mode of the metal PBG cavity. The unperturbed frequency of the mode is $2.920402 \mathrm{GHz}$. In each case the cylinder spacing was $d=3.9272 \mathrm{~cm}$, and cylinder radius was $a=0.73635 \mathrm{~cm}$. For the misalignment 
calculations the cylinders were randomly displaced by $\pm 1.47 \mathrm{~mm}$ in the $x$ and $y$ directions. This translation corresponded to the distance between points on the discretization grid.

\begin{tabular}{|c|c|c|c|}
\hline $\begin{array}{c}\text { Fundamental } \\
\text { Parameter }\end{array}$ & $\begin{array}{l}\text { TESLA (HERA) } \\
\text { Cavity }\end{array}$ & $\begin{array}{c}\text { Scaled TESLA } \\
\text { Cavity }\end{array}$ & $\begin{array}{c}\text { A } 7 \times 7 \text { PBG } \\
\text { Cavity }\end{array}$ \\
\hline $\begin{array}{l}\text { Lattice Width d } \\
\text { (mm) }\end{array}$ & N/A & N/A & 41.999 \\
\hline $\begin{array}{c}\text { Cylinder Radius a } \\
\text { (mm) }\end{array}$ & N/A & N/A & 7.875 \\
\hline $\begin{array}{c}\text { Beam Hole Radius } \\
\qquad \mathrm{rb}(\mathrm{mm})\end{array}$ & 35.0 & 15.918 & 15.919 \\
\hline $\begin{array}{c}\text { Nose Radius } r_{n} \\
(\mathrm{~mm})\end{array}$ & 12.0 & 5.458 & 5.458 \\
\hline $\begin{array}{l}\text { Half Gap Length for } \\
\pi \text {-Mode, } L \\
\text { (mm) }\end{array}$ & 57.692 & 26.238 & $\begin{array}{c}26.242 \\
\text { (includes two } \\
\text { plates; } \\
\text { thickness } 2 \mathrm{xr}_{\mathrm{n}} \text { ) }\end{array}$ \\
\hline $\begin{array}{c}\text { Fundamental } \pi \text {-Mode } \\
\text { Frequency } \\
(\mathrm{GHz})\end{array}$ & 1.299 & 2.8560 & 2.8575 \\
\hline $\begin{array}{l}\text { Peak Electric Field } \\
\text { to Acceleration Field } \\
\text { Ratio Epeak/Eacc }\end{array}$ & $2.39 \cdot$ & 2.39 & 2.58 \\
\hline $\begin{array}{c}\text { Peak Magnetic Field } \\
\text { to Acceleration Field } \\
\text { Ratio } \mathrm{H}_{\text {peak }} / \mathrm{E}_{\mathrm{acc}} \\
{[\mathrm{Oe} /(\mathrm{MV} / \mathrm{m})]} \\
\end{array}$ & 46.36 & 46.36 & 104.22 \\
\hline $\begin{array}{c}\text { Total Stored Energy } \\
\mathrm{U}_{0} / \mathrm{E}_{\mathrm{acc}}{ }^{2} \\
{\left[\mathrm{~J} /(\mathrm{MV} / \mathrm{m})^{2}\right]}\end{array}$ & 0.0141 & $1.335 \times 10^{-3}$ & $1.350 \times 10^{-3}$ \\
\hline $\begin{array}{l}\text { Q0 Factor (copper, } \\
\text { room temperature) }\end{array}$ & $26,879.6$ & $18,125.0$ & $14,790.8$ \\
\hline $\begin{array}{c}\text { Shunt Impedance R } \\
(\mathrm{M} \Omega)\end{array}$ & 3.074 & 2.073 & 1.681 \\
\hline $\mathrm{R} / \mathrm{QO}(\Omega)$ & 114.4 & 114.4 & 113.7 \\
\hline Geometry Factor G & 252.7 & 252.7 & 206.3 \\
\hline $\begin{array}{l}\text { Loss Parameter to } \\
\text { Fundamental Mode } \\
\text { kfu (Beam Bunch } \\
\sigma=6 \mathrm{~mm})(\mathrm{V} / \mathrm{pC})\end{array}$ & 0.2305 & 0.4811 & 0.4784 \\
\hline
\end{tabular}

FIGURE 5. MAFIA simulation comparison of parameters for the TESLA, Scaled Tesla, and $7 \times 7$ PBG cavities. Note that we assume the cavities are made of copper at room temperature. 


\section{SUPERCONDUCTING TEST CAVITIES}

Encouraged by early numerical simulation results, as well as initial tests on copper PBG cavities at room temperature, we designed and had fabricated the first set of superconducting (SC) PBG cavities. The SC-PBG cavities consisted of a periodic array of $\mathrm{Nb}$ cylinders, forming the underlying photonic lattice, bounded on top and bottm by $\mathrm{Nb}$ plates. These first $\mathrm{SC}$ cavities were desgned to resonate at $\sim 11 \mathrm{GHz}$ so that they would be small enough for $Q$ measurements and low-power testing in a small liquidhelium dewar. The experience gained in the fabrication and welding techniques, however, are directly applicable to the final high power test structure, which will be at $2.856 \mathrm{GHz}$ in order to be compatible with the available if power system at the Accelerator Test Facility at Brookhaven National Laboratory.

The foremost consideration in constructing the SC cavity is to determine the optimal method of joining the many cylinders of the photonic lattice to the bounding plates, since the ultimate $Q$ of the cavity in practice may be set by the surface resistance for current flow at these points. The initial approach was to use electron-beam welding to join the $\mathrm{Nb}$ cylinders to the $\mathrm{Nb}$ plates, which was performed at CEBAF. For a first test, a $3 \times 3$ cavity was constructed. The CEBAF shop made a total of 16 weld joints, varying settings on the e-beam welder to optimize welding conditions. As a result of this first test, the settings required to produce clean, reproducible welds were determined.

In order to perform low power testing and Q measurements of the Nb PBG test cavity, a small liquid helium test dewar was assembled, which operates in the frequency range of $1-20 \mathrm{GHz}$ and over the temperature range of pumped helium to room temperature. The testing unit consists of a support structure for mounting the PBG cavity which resides inside of a commercial liquid helium dewar.

After electron beam welding by the CEBAF group, the $7 \times 7$ cavity, with no absorber, was tested from room temperature down to $4.2 \mathrm{~K}$, and the cavity $\mathrm{Q}$ measured at selected temperatures. At $4.8 \mathrm{~K}$, the measured $\mathrm{Q}$ of the $7 \times 7$ cavity was found to be at least $1.2 \times 10^{6}$. This $\mathrm{Q}$ was determined by analyzing the reflected power spectrum over the frequency range of the resonance, and calculating the FWHM of the resonance curve. The cavity was strongly under-coupled for the measurement, such that the measured $Q$ 'should be close to the unloaded cavity $Q$. This measurement provides a lower limit for what the cavity $Q$ actually is, since the resolution of our test equipment places a limit on the $Q$ we can measure. For more accurate determination of such high $Q$ 's, an energy decay measurement will be used.

\section{CONCLUSION}

We are continuing our numerical exploration of the characteristics of the PBG cavity. The extensive modeling done so far has revealed both the potential advantages and weaknesses of the PBG cavity relative to current and proposed accelerator cavities. We continue to be hopeful about the prospect of producing a PBG cavity with welldamped HOMs; indeed, for room-temperature copper structures, we believe that we have succeeded in that goal. The reduction of these HOMs indicates the PBG cavity may find use in high-current applications; furthermore, the ability to alter the HOMs without 
affecting the fundamental suggests that the PBG cavity might also be a potential candidate for a detuned structure, such as the SLAC DDS.

While we remain optimistic with respect to developing high-gradient structures, it is clear from the simulations that it will be necessary to find a geometry in which $\mathrm{H}_{\text {peak }} / \mathrm{E}_{\mathrm{acc}}$ can be reduced (see Figure 5). For superconducting applications, this appears to be the most limiting factor for PBG cavities. One potential method of doing this would be to adjust the lattice parameters such that the fundamental would be less localized to the interior, allowing the confining current to be shared throughout more of the cylinders. This would reduce $\mathrm{H}_{\text {peak }} / \mathrm{E}_{\mathrm{acc}}$, but at the expense of also reducing the shunt impedance, and potentially requiring more layers of cylinders. Nevertheless, such design modifications can be made, and the feasibility of the resulting structure will depend on the application and competitive cavities.

Finally, we note that the PBG structure is relatively simple to construct, requiring only rod and flat sheets. We feel that fabrication of these structures could be implemented with current technologies at frequencies as high as $90 \mathrm{GHz}$. Furthermore, substituting dielectrics for any or all of the metal rods also produces a PBG structure which can have few (possibly one) localized modes. Previous work (4) suggests that suitably treated dielectrics can exhibit very low loss, and are capable of sustaining large gradients; thus, we intend to further investigate both pure dielectric structures, as well as dielectric/metal hybrid structures.

\section{ACKNOWLEDGEMENTS}

We thank Ricci Campisi and John Brawley for their asssistance in fabrication of the superconducting PBG cavities, and AccSys Technologies for fabrication of the copper test cavities used in this study. We also thank $\mathrm{H}$. Padamsee for helpful discussions. This research has been supported by the DOE, contracts DE-FG-0393ER40793 and DE-AC-03-76SF00515.

\section{REFERENCES}

1. Schultz S., Smith D. R., and Kroll N., Proc. 1993 Particle Accelerator Conf, (May 1993 Washington, D. C.) IEEE, 4, 2559 (1994)

2. Thompson K. A., Adolphsen C., Bane K. L. F., Deruyter H., Farkas Z. D., et. al., Particle Acclerators, 47, 65 (1994)

3. Kroll N., Thompson K., Bane K., Gluckstern R., Ko K., Miller R., and Ruth R., 6th Workshop on Advanced Accelerator Concepts, Lake Geneva, WI, June 12-18 (1994); SLAC-PUB-6660

4. Gai W., Schoessow P., Cole B., Konecny R., Norem J., Rosenzweig J., and Simpson J., Phys. Rev. Lett., 61, 2756 (1988) 